\title{
Welche Frage, wann und warum? - Eine qualitativ-linguistische Programmatik zur Erforschung von Frage-Sequenzen als zentrale Veränderungspraktik im Coaching
}

\author{
Eva-Maria Graf' ${ }^{1}$ · Thomas Spranz-Fogasy² \\ Online publiziert: 18. Januar 2018 \\ (c) Der/die Autor(en) 2018. Dieser Artikel ist eine Open-Access-Publikation.
}

\section{Zusammenfassung}

Der folgende Beitrag fokussiert die kommunikative Praktik „Fragen“ im Beratungsformat Führungskräfte-Coaching. Fragen stellen laut Praxis-Literatur und Ausbildungsmanualen zu Coaching ein, wenn nicht das, zentrale Interventionsinstrumentarium dar. Trotz dieser formulierten Omnipräsenz und Omnirelevanz gibt es bis dato kaum empirische Erkenntnisse über die tatsächliche Verwendung von Fragen im Coaching. Fragen sind weder in der quantitativ operierenden, psychologischen Wirksamkeits- bzw. Outcome-Forschung noch in der qualitativ operierenden, linguistischen Prozessforschung (zentraler) Forschungsgegenstand. Diese Forschungslücke gilt es im Austausch mit der Praxis und unter Einbezug aller relevanten Disziplinen und Methoden zu schließen. In einem ersten vorbereitenden Schritt macht es sich der vorliegende programmatische Beitrag zur Aufgabe, das Phänomen „Fragen im Coaching“ als Forschungsgegenstand der linguistischen Gesprächsanalyse zu etablieren. Fragen im Coaching werden dabei sowohl bezüglich ihrer Form, ihrer Funktion als auch als institutionsspezifische soziale Praktik diskutiert, wobei Erkenntnisse zur Verwendung von Fragen in benachbarten professionellen Gesprächen wie Psychotherapie oder Arzt-Patient-Kommunikation als erste Orientierung herangezogen werden. Im Zentrum der gesprächsanalytischen Diskussion steht der Beitrag, den Frage-Sequenzen zur Veränderung und damit zur lokalen Wirksamkeit von Coaching leisten. Der Artikel endet mit einer kritischen Evaluation der Möglichkeiten einer gesprächsanalytischen Erforschung von Frage-Sequenzen und skizziert den Mehrwert von interprofessioneller und interdisziplinärer, insbesondere linguistischer und psychologischer, Forschung für die Coaching-Praxis.

Schlüsselwörter Frage-Sequenzen · Coaching-Gespräche · Sequentielle Ko-Konstruktion · Wirksamkeit · Linguistische Gesprächsanalyse $\cdot$ Interprofessionalität $\cdot$ Interdisziplinarität

Publisher's Note Springer Nature remains neutral with regard to jurisdictional claims in published maps and institutional affiliations.

Eva-Maria Graf

Eva-Maria.Graf@aau.at

1 Institut für Anglistik und Amerikanistik, Alpen-Adria-Universität Klagenfurt, Klagenfurt, Österreich

2 Institut für Deutsche Sprache, Mannheim, Deutschland 


\title{
Which Question, When and Why? - A Qualitative-Linguistic Programmatic to Analyze Question Sequences as a Principal Change Practice in Coaching
}

\begin{abstract}
Questions symbolize an important, if not the most important intervention tool in coaching according to the coaching literature and training manuals. Despite such claimed omnipresence and omnirelevance in coaching theory, there are hardly any empirical findings to back up such claims. Neither in quantitatively operating psychological effectiveness-research nor in qualitatively operating linguistic or psychological process-research on coaching questions have received much empirical attention. It is vital to close this research gap via a cooperation between coaching practice and relevant disciplines and methods. In a first preliminary step, this programmatic paper establishes "questions in coaching" as a proper research object in conversation analysis. Questions are discussed in their formal and functional dimensions as well as an institution-specific social practice. Conversation-analytic findings from surrounding professional formats such as psychotherapy or doctor-patient interaction serve as points of orientation. The linguistic and CA discussion focusses on the impact of question-sequences on change and thus on the efficiency of coaching. The article ends with a critical evaluation of a conversation analytic approach to question sequences in coaching and sketches out the added value (for the coaching practice) of an interprofessional and interdisciplinary research that draws on coaching theory as well as linguistic and psychological methods and theories.
\end{abstract}

Keywords Question-sequences · coaching conversation · Sequential co-construction · Efficiency · Conversation analysis · Interprofessionality $\cdot$ Interdisciplinarity

\section{Fragen als kommunikative Praktik' im Führungskräfte-Coaching}

Fragen sind sowohl in Alltagsgesprächen als auch in professionellen und institutionellen Gesprächen ein zentrales und allgegenwärtiges Steuerungsmittel (vgl. Tsui 1992; Freed und Ehrlich 2010; Heritage 2010; Hayano 2013; Drew und Couper-Kuhlen 2014) ${ }^{2}$. Fragen gehen dabei auf eine elementare anthropologische Fähigkeit des Menschen zurück, die Köller (2004, S. 662) folgendermaßen zusammenfasst:

Fragen leiten hypothetische Vorstellungsprozesse ein, die eine immanente Tendenz haben, in Selbstreflexionsprozesse überzugehen. Fragen helfen uns, Wissensdefizite zu lokalisieren, Wissensbedürfnisse zu thematisieren, Interessen für Ursachen und Funktionen zu artikulieren, Bezüge zur Vergangenheit sowie Zukunft herzustellen (...) Fragen setzen einerseits immer Erfahrungen voraus, sie sind andererseits aber auch dazu bestimmt, neue Erfahrungen zu ermöglichen, weil sie einen Wechsel von Sehepunkten und Wahrnehmungsperspektiven beinhalten.

Diese von Köller skizzierten Funktionen bzw. Aufgaben von Fragen gelten dabei in besonderer Art und Weise auch für Fragen im professionellen Gesprächstyp „Führungskräf-

\footnotetext{
${ }^{1}$ Für eine aktuelle und umfassende Diskussion des Begriffs bzw. Konzepts der Praktik siehe Deppermann et al. (2016).

2 Siehe auch das special issue des Journal of Pragmatics (42) 2010 zum Thema Question-Response Sequences in Conversation across Ten Languages.
}

te-Coaching“. Sich auf Fragen im Coaching zu fokussieren, bedeutet sich einer wesentlichen, weil zur Reflexion anregenden, Veränderung induzierenden Praktik im Kontext professioneller Beratungsgespräche $\mathrm{zu}$ widmen (Bercelli et al. 2008; Stokoe und Sikveland 2016): „Adequate reflection on one's experience is often seen as a stepping stone to change because reflection can allow the client to construe his or her life and social relationships in additional and alternative ways" (Muntigl und Zabala 2008, S. 188). In ihrer endemischen Sequenzialität als adjacency pair, also des handlungslogischen Zug-um-Zug-Verlaufs des Coaching-Gesprächs im Kontext von Frage-AntwortSequenzen, sind sie paradigmatisch für das Diktum der lokalen Wirksamkeit, d.h. für die Ko-Konstruktion von neuem Wissen für die Klient_innen auf der Mikroebene des Gesprächs: Veränderung wird erst durch die sequenzielle Organisation von helfenden Gesprächen wie Therapie ermöglicht, da kommunikative Praktiken wie Fragen sich an eine bestimmte Person richten, eine thematische Richtung des Gesprächs vorgeben sowie eine bestimmte Art der sprachlichen Reaktion im Gegenüber konditional relevant setzen (Peräkylä 2011, 2013) .

Zunächst soll hier kurz der interaktive und kommunikative Rahmen für den Einsatz von Fragen skizziert werden. Diesem programmatischen Beitrag und der linguis-

\footnotetext{
3 Vergleiche hierzu auch Voutilainen et al. (2011, S. 348): ,an analysis that focuses on sequences of talk that are interactionally similar offers a sensitive method to investigate the manifestation of therapeutic change.“.
} 
tisch-gesprächsanalytischen ${ }^{4}$ Erforschung von Fragen liegen ein dialogisches Verständnis von Coaching zugrunde: Coaching ist ein Gespräch bzw. eine kommunikative Interaktion, die von den Teilnehmer_innen, also Coach und Klient_in, lokal im Hier und Jetzt ihrer Begegnung gemeinsam hergestellt, konstituiert wird (Deppermann, 2008) (siehe Graf 2015, in Vorbereitung). Das Coaching-Gespräch als interaktiver Prozess fokussiert somit neben den Coaches immer notwendigerweise auch die Klient_innen, und basiert auf situativ gesteuerten, intentionalen und wechselseitigen Verständigungshandlungen. Eine monologische Sichtweise auf Coaching, die nur die Handlungen des Coaches, z. B. ausschließlich das Stellen von Fragen, nicht aber die Reaktionen darauf berücksichtigt, kann keine befriedigende Auskunft über eine tatsächliche Verwendung kommunikativer Praktiken wie Fragen geben ${ }^{5}$. Die Sequenzialität von Gesprächen ist ein zentrales Axiom des sprachlichen Handelns, die Auer (1999, S. 138) in Anlehnung an Harvey Sacks, den Begründer der ethnomethodologischen Konversationsanalyse, so erklärt: ,,... wir erkennen aus der Art und Weise, wie der andere seine nächsten Handlungen formuliert, wie er unsere erste Handlung verstanden hat" (siehe auch ausführlich Schegloff 2007; Stivers 2013). Neben solch prospektiver Einbettung der einzelnen Äußerungen bedeutet Sequenzialität im Gespräch aber auch notwendigerweise den retrospektiven Bezug einzelner Äußerungen zu dem, was ihnen (unmittelbar) vorausgeht: „Turn-constructional units, and the actions produced with them, are proffered by reference to what came before and affect what comes next" (Stivers 2013, S. 191). Oder wie Tiittula (2001, S. 1362) formuliert: „Initiierende Aspekte projizieren relevante Folgeaktivitäten, respondierende Aspekte verweisen zurück; die erstgenannten sind kontextdeterminierend, die letztgenannten kontextdeterminiert". Vor dem Hintergrund eines interaktionstheoretischen und gesprächsanalytischen Verständnisses von Coaching als Gespräch erfordert die Analyse kommunikativer Handlungen wie Fragen somit immer (minimal) den Einbezug des vorausgehenden und des nachfolgenden Redebeitrags, wobei die Gestaltung der Re-

\footnotetext{
${ }^{4}$ Die (linguistische) Gesprächsanalyse stellt einen zentralen Forschungsansatz innerhalb der Linguistik dar, der sich der Analyse von professionellen und alltäglichen Gesprächen und ihren interaktiven und kommunikativen Charakteristika widmet. Sie ist dabei als deutschsprachige Forschungstradition mit der aus den USA stammenden Conversation Analysis (= Konversationsanalyse; Sidnell und Stivers 2013) verwandt, aber nicht deckungsgleich und bildet den theoretisch-methodischen Rahmen dieses Beitrags. Wird von Linguistik zw. linguistisch gesprochen ist die gesamte Wissenschaft gemeint im Unterschied z. B. zur Psychologie.

5 Für eine gesprächsanalytische Herangehensweise bedeutet dies, dass der Fokus auf der sequenziellen Einbettung von Fragen im Coaching liegt, d. h. die Antworten der Beteiligten werden stets mitberücksichtigt (vgl. Lee 2013). Statt von Fragen soll deshalb von Frage-Sequenzen gesprochen werden (siehe auch Stivers und Enfield 2010).
}

debeiträge neben dem individuellen auch vom jeweiligen Interaktionstyp und sozialen Kontext geprägt ist. Aus gesprächsanalytischer Sicht ist hierbei relevant, inwieweit es sich bezüglich des sozialen Kontextes um Alltagsgespräche oder um professionelle und/oder institutionelle Gespräche handelt (Drew und Heritage 1992; Schnurr 2013): Der soziale Kontext „professionelles und/oder institutionelles Gespräch“ unterscheidet sich vom sozialen Kontext „Alltagsgespräch“ durch unterschiedliche Verantwortlichkeiten, Beteiligungsvoraussetzungen und Wissensstände der Beteiligten (siehe unten). Neben der sprachlich-kommunikativen Dimension im Sinne einer dialogischen Ko-Konstruktion muss beim Coaching also auch die institutionelle Dimension des Gesprächs als Rahmen für den Einsatz bzw. die Analyse von Fragen-Sequenzen berücksichtigt werden.

Bei Coaching handelt es sich um ein professionelles Hilfe-Gespräch, wobei die Hilfe in und durch das Gespräch der Beteiligten geleistet wird (vgl. Miller und Considine 2009; Graf et al. (eds.) 2014), d.h., das sprachliche Handeln der Beteiligten realisiert den Zweck der Institution Coaching (vgl. Pawelczyk 2011 und Scarvaglieri 2013 für Psychotherapie; Mack et al. 2016 für OPD; Nowak 2010 und Menz et al. 2010 für Medizin). Innerhalb der helfenden Gespräche handelt es sich bei Coaching um ein spezifisches Beratungsformat im beruflichen Kontext (vgl. Kallmeyer 2001; Graf 2017; Pick 2017); im Sinne einer Prozessberatung im Unterschied zur klassischen Experten-Beratung unterstützt Coaching Klient_innen dabei, eigene Antworten auf und Lösungen für ihre Anliegen sprachlich-kommunikativ zu erarbeiten. In dieser (idealisierten) Hilfe-zur-Selbst-Hilfe ist Wissenskonstitution und -umstrukturierung in und durch das Gespräch neben der Beziehungsgestaltung der instrumentelle Zweck des Interaktionstyps (Spranz-Fogasy und Lindtner 2009; Miller und Considine 2009; Spranz-Fogasy und Graf in Vorbereitung a). Wissen als Teilnehmerkonstrukt und als Ressource der Interaktionsorganisation (vgl. Deppermann 2015, S. 4) weist dabei je nach Beratungstyp spezifische (kommunikative und interaktive) Formen auf und ist nicht gleich unter den Gesprächsteilnehmer_innen verteilt, ,Wissensasymmetrien sind eine wesentliche Determinante sozialer Beziehungen“" (Deppermann 2015, S. 12). So verfügen Berater_innen über theoretisches Erkenntniswissen, während Klient_innen über subjektives Erfahrungswissen bezüglich ihres Anliegens verfügen (Sarangi 2001). In professionellen (Beratungs-)Gesprächen konstituiert diese Wissensasymmetrie zwischen Berater_in und zu beratender Person, die sich daraus ergebende unterschiedliche Problemlösekompetenz sowie der Perspektivenunterschied zwischen z.B. Coach und Klient_in, den eigentlichen Beratungsbedarf (Kallmeyer 2001).

Die Unterscheidung in Expertenberatung und Prozessberatung als eigenständige Beratungskonzepte (Schein 1969; Schreyögg 2010; Geißler 2013) fokussiert (primär) die Phä- 
nomene Wissensvermittlung und Wissensgenerierung. Da Wissen in und durch das jeweilige (Beratungs-)Gespräch vermittelt und generiert wird, manifestieren sich die Beratungstypen sprachlich-kommunikativ auf der Ebene der Gesprächsführung im Allgemeinen und in spezifischen Fragepraktiken (und anderen Praktiken wie Reformulierungen und Interpretationen) als Bestandteile der Gesprächsführung im Besonderen. So hat Balint schon 1957 für die ArztPatient-Kommunikation in krankheitszentrierte und patientenorientierte Gesprächsführung unterschieden (vgl. auch Lalouschek 2005; Spranz-Fogasy 2005); letztere findet sich im psychotherapeutischen Kontext wieder in Carl Rogers' klientenzentrierter Therapie (1951), die als Vorbild für die Gesprächsführung im Coaching gesehen wird (vgl. Schein 1969). Gesprächsführung wird hier ganz allgemein verstanden als der Einsatz von Formulierungen, Ablaufformen und institutionellen kommunikativen Mustern sowie ihre konkreten und lokalen Formen, Funktionen und Wirkungen im Gespräch (Spranz-Fogasy 1992; Mönnich 2004); stärker im Zusammenhang mit Steuerung, Dominanz und Asymmetrie verstanden definiert Tiittula (2001) Gesprächssteuerung bzw. gesprächssteuernde Aktivitäten als solche, mit denen die Interagierenden auf den Verlauf des Gesprächs lenkend einwirken, in dem sie z.B. regeln, wer das Rederecht erhält, worüber gesprochen wird und welche Handlungen die Gesprächspartner_innen durchzuführen haben (ibid, S. 1361). Fragen sind somit zentrale Steuerungselemente, da sie sich an eine bestimmte Person richten, inhaltlich eine thematische Richtung vorgeben und darüber hinaus eine bestimmte Art der Erwiderung mehr oder weniger stark konditionell ${ }^{6}$ relevant setzen. Bezüglich der oben aufgeführten Unterscheidung in Alltagsgespräche und professionelle und/oder institutionelle Gespräche geht Tiittula (2001, S. 1362) davon aus, dass ,,(d)ie Steuerungsmöglichkeiten variieren je nach Situations- und Ereignistyp und den damit verbundenen Beteiligungsrollen“. In Winklers (2017) gesprächsanalytischer Untersuchung von (Chat-)Coaching wird dabei konkretisierend die Gesprächssteuerung durch den Coach auf der Ebene der Gesprächsorganisation, der Ebene der Themenkonstitution sowie auf der Ebene der Handlungskonstitution als entscheidendes Kriterium einer gelingenden Gesprächsführung verstanden. Die Gesprächsführung der professionell Agierenden ist dabei im Sinne einer institutionellen Rahmung geprägt von den dem Coaching-Gespräch zugrundeliegenden professionellen Agenden, die das Erreichen der professionellen Ziele ermöglichen (vgl. Peräkylä und Vehviläinens (2003) professional stocks of inter-

\footnotetext{
${ }^{6}$ Ist eine fokale $\ddot{A} u ß e r u n g$ (z. B. eine Frage) dazu bestimmt, eine spezifische Reaktion (z.B. eine Antwort) hervorzurufen, wird diese Eigenschaft als konditionelle Relevanz bezeichnet: Die erste Äußerung schafft Bedingungen, durch die ein bestimmter Typ von Folgehandlungen relevant wird. (Deppermann 2008, S. 68).
}

action knowledge). Diese Agenden prädeterminieren mehr oder weniger grob den Gesprächsverlauf in seiner internen Aufgaben- und Handlungslogik, wobei die Einhaltung desselben in der Macht der professionell Agierenden, d.h. der Coaches, liegt. Systematische Asymmetrien und unterschiedliche Dominanz ${ }^{7}$ im Gespräch sind somit endemische Bestandteile professioneller Gespräche (vgl. Tiittula 2001, S. 1362$)^{8}$.

Unterschiedliche kommunikative Praktiken der Gesprächsführung wie (Re-)Formulierungen, Interpretationen oder Fragen bzw. verschiedene Fragetypen weisen dabei im Gespräch wiederum eine unterschiedliche Interventionsstärke auf; ihr Einsatz dominiert die Gesprächspartner_innen, in unterschiedlichem Ausmaß bzw. durch ihren Einsatz werden sie unterschiedlich stark geführt bzw. gesteuert. Um mit Steensig und Drew (2008, S. 7) konkret auf die hier im Zentrum stehende kommunikative Praktik der Fragen Bezug zu nehmen, kann man sagen, dass, ,,(a)sking a question is not an innocent thing to do". Vielmehr können Coaches über Fragen sowohl den gesprächsorganisatorischen Ablauf der Interaktion, d.h. der CoachingSitzung, kontrollieren, in dem sie durch das Stellen von Fragen bestimmte Folgehandlungen konditional relevant setzen, als auch den inhaltlichen bzw. thematischen $\mathrm{Ab}$ lauf der Interaktion beeinflussen bzw. kontrollieren in dem sie unterschiedliche thematische Aspekte (aus den Erzählungen der Gesprächspartner_innen) relevant setzen (vgl. Lalouschek 2002, S. 162). Dass sich die verschiedenen professionellen Konzepte der Gesprächsführung in der Verwendung unterschiedlicher Fragetypen manifestieren, wies z.B. Spranz-Fogasy (2005) im Zusammenhang mit Präzisierungsfragen vs. Komplettierungsfragen im Kontext medizinischer Kommunikation nach: das patientengeleitete Konzept arbeitet primär mit Präzisierungsfragen, also Fragen, die an das Wissen der Patient_innen anschließen, während das krankheitszentrierte oder wissensgeleitete Konzept primär mit Komplettierungsfragen, also Fragen, die sich am Wissen der Ärzte und Ärztinnen orientieren, arbeitet (für Details zu den Fragetypen siehe Punkt 3).

Zusammenfassend basiert eine gesprächsanalytische Annäherung an Fragen-Sequenzen im FührungskräfteCoaching auf folgenden kommunikativen und interaktionstheoretischen Axiomen:

\footnotetext{
7 Asymmetrie, Steuerung, Dominanz und Hierarchie sind eng miteinander verbunden, vgl. hierzu z. B. Brock und Meer (2004).

${ }^{8}$ Die in der Praxisliteratur zu Coaching wiederholte Aufteilung der Verantwortung, d.h. Coaches sind für den Prozess, Klient_innen sind für die Inhalte verantwortlich, findet sich hier teilweise bestätigt; jedoch steuern Coaches über Fragen auch den inhaltlichen Verlauf der Gespräche.
} 
1. Coaching wird in und durch das miteinander Sprechen der Beteiligten lokal und dialogisch im Hier-und-Jetzt hergestellt; Coaching realisiert sich in einem Gespräch.

2. In diesem Gespräch stehen die Gesprächsbeiträge jeweils wechselseitig mit dem vorher Gesagten in gesprächsorganisatorischer und thematischer Verbindung und bilden prospektiv für den folgenden Beitrag gesprächsorganisatorisch und thematisch die Voraussetzung; im Sinne dieser Sequenzialität sind initiierende Beiträge dabei kontextdeterminierend, respondierende Beiträge sind kontextdeterminiert.

3. Das Coaching-Gespräch zeichnet sich als professionelles Gespräch durch unterschiedliche Beteiligungsvoraussetzungen, Verantwortlichkeiten und Wissensbestände der Beteiligten aus; diese manifestieren sich auf der Ebene der Gesprächsführung in unterschiedlichen Steuerungsmöglichkeiten durch Elemente wie Fragen sowie in unterschiedlichen Realisierungsformen von Asymmetrie und Dominanz.

4. Als professionelles Gespräch stellt Coaching eine helfende Interaktion bzw. ein Beratungsgespräch dar, in dem Wissensvermittlung und Wissensgenerierung die sine qua non für das Zustandekommen der helfenden Interaktion sind.

5. Wissensvermittlung und Wissensgenerierung als zentrale Elemente der professionellen Interaktion Coaching prägen die professionelle Agenda der Coaches und die spezielle Gesprächsführung, wobei im Coaching im Sinne einer Prozessberatung, im Unterschied zur Expertenberatung, (idealisiert) von einer personenorientierten Gesprächsführung auszugehen ist ${ }^{9}$.

6. Die Gesprächsführung beinhaltet neben anderen Steuerungselementen die kommunikative Praktik des Fragens, die - im Sinne des zweiten Axioms der Sequenzialität - nicht monologisch als singulärer Sprechakt, sondern dialogisch als Redebeitrag retrospektiv und prospektiv in den Gesprächsverlauf eingebettet ist.

7. Durch kommunikative Praktiken wie Fragen steuern die professionell Agierenden, z. B. die Coaches, den Verlauf des Coachings sowohl inhaltlich als auch gesprächsorganisatorisch.

8. Gleichzeitig garantiert und ermöglicht die sequenzielle Organisation von Gesprächen, die u.a. paradigmatisch durch Frage-Sequenzen hervorgebracht wird, ihre lokale Wirksamkeit.

Diese Axiome leiten das gesprächsanalytische Vorgehen zur Erforschung von Fragen im Coaching. Das konkrete Forschungsinteresse und die Motivation für den vorliegen-

\footnotetext{
${ }^{9}$ Inwieweit diese Art der Gesprächsführung das tatsächliche Coaching-Gespräch prägt oder es sich um eine idealisierte Darstellung der Praxis-Literatur handelt, ist eine noch offene Forschungsfrage.
}

den Beitrag ${ }^{10}$ resultiert aus der Diskrepanz zwischen der Omnipräsenz von Fragen in Praxisliteratur und Lehrbüchern zu Coaching und der unbefriedigenden Forschungssituation im Zusammenhang mit Fragen. Obwohl erste empirische Ergebnisse zeigen, dass Fragen rein quantitativ ein zentrales Steuerungselement im Coaching sind (vgl. Deplazes 2016), liegen noch keine weiterführenden Erkenntnisse bezüglich der Funktionen von Frage-Sequenzen für die Etablierung Coaching-relevanter Inhalte, der Durcharbeitung dieser Inhalte sowie zur Etablierung und Durchführung der Handlungslogik gemäß der Bearbeitung der den Coaching-Prozess formenden kommunikativen Basisaktivitäten ${ }^{11}$ (Graf 2015, in Vorbereitung) vor.

Der Beitrag ist wie folgt strukturiert: Zunächst wird die Darstellung von Fragen in der Coaching-Praxisliteratur und den Lehrbüchern sowie die (lückenhafte) Beschäftigung mit Fragen in der Coaching-Forschung skizziert (Abschn. 2). Darauf aufbauend werden Fragen aus linguistisch-gesprächsanalytischer Sicht sowohl bezüglich ihrer Form und Funktion als auch im Sinne einer institutionellen Praktik (Tracy und Robles 2009) dargestellt (Abschn. 3). Erkenntnisse zu Fragen aus benachbarten professionellen Formaten wie Psychotherapie oder Arzt-Patient-Kommunikation stützen diese Darstellung, da zu Coaching noch keinerlei gesprächsanalytische Ergebnisse vorliegen. Es ist allerdings davon auszugehen, dass die $\mathrm{zu}$ entwickelnde Typologie von Fragen-Sequenzen im Coaching format-spezifische Charakteristika aufweist, da Fragen immer auch - neben formalen und funktionalen Aspekten - interaktionstypspezifisch endemische Charakteristika aufweisen. Im Ausblick, Abschn. 4, werden die Grenzen einer rein gesprächsanalytischen Herangehensweise aufgezeigt, der Mehrwert einer interprofessionellen und interdisziplinären Erforschung von Fragen-Sequenzen diskutiert, vor allem vor dem Hintergrund der angestrebten Praxisrelevanz solcher empirischen Erkenntnisse für den Coaching-Weiter-

\footnotetext{
${ }^{10}$ Darüber hinaus ist ein interprofessionelles und interdisziplinäres Forschungsprojekt in Planung (Graf et al. in Vorbereitung), das sich der systematischen Beschreibung und Typologisierung von FrageSequenzen als zentraler Praktik der Gesprächsführung im Coaching widmet.

${ }^{11}$ Ein zentrales Ergebnis der gesprächsanalytischen Coaching-Prozessforschung ist (Graf 2015, in Vorbereitung), dass sich sowohl der gesamte Coaching-Prozess als auch die einzelnen Sitzungen schleifenartig in und durch sich wiederholende und ineinander verwobene kommunikativen Aktivitäten vollziehen: mit Hilfe der Analyse authentischer Coaching-Gespräche wurde anstelle eines Phasenmodells wie in der Praxisliteratur proklamiert das Modell der kommunikativen Basisaktivitäten, bestehend aus den vier Aktivitäten „Definieren der Situation“, „Gestalten der Beziehung“, „Ko-Konstruieren der Veränderung“ und „Evaluieren des Coachings“ entwickelt. Die einzelnen Aktivitäten bestehen jeweils aus wiederkehrenden kommunikativen Aufgaben, zu deren Lösung den Beteiligten bestimmte diskursive Praktiken und konkrete sprachliche Realisierungen zu Verfügung stehen.
} 
bildungsmarkt. Im Sinne eines theoretischen Grundlagenartikels werden im Beitrag keine empirischen Ergebnisse präsentiert.

\section{Fragen im Coaching im Spiegel der Praxisliteratur und Praxisforschung}

Was für den Geiger die Geige ist, ist für den Coach die Frage: ein Instrument, ohne das er seinen Beruf nicht ausüben kann (Wehrle 2012, S. 7).

Das angeführte Zitat von Wehrle (2012) aus seinem Werk „Die 500 besten Coaching-Fragen“ illustriert stellvertretend die in der existierenden Praxisliteratur reklamierte Zentralität von Fragen für Coaching bzw. Coaches. Liest man die Praxisliteratur bzw. die Lehrbücher zu Coaching, so entsteht der Eindruck als wäre ,gutes“ und „erfolgreiches“ Coaching gleichzusetzen damit, als Coach möglichst viele Fragen zu stellen und dabei eine möglichst große Bandbreite an Fragetypen einzusetzen: Wilmes und Loebbert (2013, S. 38) sprechen bei Fragen vom „Königsweg zur Exploration des Klienten“, Fischer-Epe (2012, S. 60) argumentiert, dass Fragen eine ,zentrale Steuerungsmöglichkeit im Coaching-Prozess" sei; Schreyögg (2012, S. 269) charakterisiert Fragen als die ,wichtigste Aufgabe eines Coachs". Des Weiteren findet sich fast durchgängig die Aufforderung darauf zu achten, offene, aber keine ,geschlossenen“ Fragen zu stellen, da letztere gesprächsschließend seien und den Klient_innen keine Möglichkeit zur Reflexion ließen (Radatz 2003; Fischer-Eppe 2012; Lippmann 2013), während auf erstere ,,... schon automatisch detailreich geantwortet werden muss" (Schreyögg 2012, S. 270). Klassische Fragetypen, die in der Praxisliteratur wiederholt gelistet werden sind etwa „Wunderfrage“ „Hypothetische Fragen“, „Zirkuläre Fragen“, „Systemische Fragen“, „Reframing Fragen“, „Ressourcen-Fragen“, „Lösungsfragen“, „Paradoxe Fragen“, „Provokante Fragen“, „Skalierungsfragen" etc. So führen Schmidt-Tanger und Stahl (2005) z.B. 19 verschiedene Fragetypen in ihrem Werk zu Change. Coachen lernen! Coaching-Können bis zur Meisterschaft an (für eine Übersicht der Praxis und Lehrmeinung bzgl. Fragen vgl. hierzu zusammenfassend Deplazes 2016, S. 69 ff).

Die replizierte Meinung bezüglich der Wichtigkeit von Fragen in den exemplarisch diskutierten Werken basiert auf der Praxiserfahrung bzw. der persönlichen Einschätzung der Autor_innen, nicht aber auf wissenschaftlichen Erkenntnissen. Stattdessen scheint sich die Coaching-Praxis gegenseitig die Wichtigkeit von Fragen zu bestätigen und detaillierte Darstellungen in anderen Werken als Bestätigung bzw. Motivation für die eigene ausführliche Behandlung von Fragen zu nehmen. Im Kontext der Coaching-Ausbildungen, wo in den Manualen und Anleitungen Fragen eine ähnliche Relevanz gegeben wird, ist aus Sicht der Ausbildungsteilnehmer_innen eine solche Auflistung und das Vermitteln bestimmter Frage-Typen zum einen eine Hilfe, weil etwas Konkretes, das am Anfang einer Ausbildung als Handwerkszeug den Coaching-Lernenden Sicherheit geben kann. Zum anderen aber bedeutet dies auch eine Herausforderung bzw. ein Stressmoment, da Botschaften wie etwa keine geschlossenen Fragen zu stellen oder die ganze Bandbreite an Fragetypen abzudecken, bei den Teilnehmenden viel Aufmerksamkeit ausschließlich darauf bündelt.

Diese Tatsache, dass es im Coaching eine ausführliche (idealisierte) Beschreibung von richtigem Vorgehen für Praktiker_innen und Ausbildungsteilnehmer_innen gibt, ist jedoch keine Besonderheit. So formulieren Tracy and Robles (2009, S. 135) allgemein, ,(j)ust about every institutional site has a rich professional literature that offers advice to practitioners". Dabei konstatieren sie aber auch, dass konversationsanalytische Forschung immer wieder aufzeigt, dass die tatsächliche Praxis von dieser idealisierten Darstellung abweicht. Bezüglich der Verwendung von lösungsorientierten Fragen im Kontext von Mediation fanden z.B. Stokoe and Sikveland (2016, S. 103),

(h)owever, in the empirical materials, SFQs did not echo the training materials in terms of their design. Conversation analysts have regularly shown that, when practitioners attempt to turn training materials or scripted questions into actual interaction, the ensuing practices vary from those idealized versions.

Eine empirische Fundierung und Entwicklung einer Frage-Antwort-Typologie für Coaching, die auf der tatsächlichen Verwendung von Frage-Sequenzen in authentischen Coaching-Gesprächen fußt, ist somit gerade auch für die Coaching-Praxis von zentraler Bedeutung für ihr professionelles Handeln.

Im Folgenden werden zunächst die wenigen wissenschaftlichen Erkenntnisse zu Fragen im Coaching dargestellt, um dann aus der sich abzeichnenden Forschungslücke ein Forschungsdesiderat und Forschungsfragen abzuleiten.

Im Unterschied zur Zentralität des Themas „Fragen“ in Lehrbüchern und der Praxis-Literatur zu Coaching liegen in der deutsch- und englischsprachigen Coaching-Forschung bis dato kaum empirische Erkenntnisse zu Fragen vor. Erste psychologisch fundierte Ergebnis-Studien belegen zwar die Wirksamkeit von adäquaten Fragen für Coaching (vgl. Grant und O'Conner 2010; Grant 2012). Dort gewonnene allgemeine Erkenntnisse wie etwa dass „effective questioning lies at the very heart of the coaching conversation" (Grant und O'Connor 2010, S. 102), generieren aber in der psychologischen Coaching-Prozessforschung nur neue, bis dato unbeantwortete, Fragen wie etwa ,(b)ut what constitutes ,effective " questioning in coaching?" (ibid.). Allerdings berichten Grant and O'Conner (2010) in ihrer Studie mit 
australischen Studierenden auch differenzierter, dass insbesondere lösungsorientierte Fragen insgesamt effektiver sind als problemorientierte Fragen. Diese Pilot-Ergebnisse wurden 2016 von Neipp et al. (2015) mit spanischen Studierenden repliziert. Auch Braunstein und Grant (2016) sowie Theeboom et al. (2016) widmen sich der Effizienz von lösungsorientierten im Unterschied zu problemorientierten Fragen. Neben diesen vereinzelten Studien, die sich explizit und ausschließlich mit Fragen im Coaching beschäftigen, gibt es vereinzelte Forschung, die bestimmte Fragetypen neben anderen Interventionen fokussieren: so befassen sich Newson und Dent (2011) in ihrer Analyse des Coaching-Verhaltens bzw. Coaching-Handelns von Führungskräfte-Coaches u.a. mit dem Stellen von offenen Fragen und listen als Ergebnis ihrer Befragung von ca. 130 Coaches 1) establishing trust, honesty, and respect 2) using open-ended questions, and 3) clarifying and understanding client concerns and challenges (ibid, S. 18) als die am häufigsten genannten Aktivitäten. Insgesamt ist die Forschungssituation zu Fragen in der psychologisch geprägten Wirksamkeitsforschung nicht befriedigend und spiegelt die Wichtigkeit bzw. die Stellung von Fragen in der Praxisliteratur nicht wider. Umfassende Erkenntnisse zu Fragen in ihrer Vielfalt und Komplexität sind nicht zu finden. Mit ihrer psychologischen video-basierten Coaching-Prozessanalyse, die u.a. mit der qualitativen Inhaltsanalyse nach Mayring (2010) arbeitet, liefert nur Deplazes (2016) erste empirische Einblicke in „reales“ Fragen und streift dabei auch kurz die Antworten von Fragen, d.h. geht also auf die Sequenzialität von Fragen im Gespräch ein. Allerdings setzt sich die Autorin in ihrer Analyse mit allen Interventionen und Tools im Coaching auseinander, so dass die Abhandlung von Fragen notwendigerweise relativ knapp bleiben muss.

Eine ähnliche Situation findet sich in der sich neu etablierenden gesprächsanalytischen Prozess-Forschung zu Coaching: Während Fragen als relevante Gesprächshandlungen in anderen professionellen Hilfe-Formaten wie ArztPatient-Kommunikation (Spranz-Fogasy, 2005, 2010; Heritage 2010), Therapie (Mack et al. 2016) oder Beratung (Peräkylä 1995; Sarangi 2010) ${ }^{12}$ und auch insgesamt im Kontext institutioneller und professioneller Kommunikation (Freed und Ehrlich 2010) von zentralem Forschungsinteresse sind, liegen noch keinerlei Erkenntnisse über die lokale in-situ Konstruktion von Frage-Sequenzen durch Coach und Klient_in im konkreten Coaching-Gespräch vor. Dies ist sicherlich u. a. der insgesamt noch nicht umfangreichen linguistischen Coaching-Forschung geschuldet, zeigt aber doch auch hinsichtlich linguistischer Erforschung von Coa-

\footnotetext{
12 Allerdings weisen Scarvaglieri (2013, S. 42) und auch Konerding (2015, S. 229) darauf hin, dass Fragen auch in der linguistisch-gesprächsanalytischen Forschung zu psychotherapeutischen Handlungen bisher eher wenig Beachtung erfahren haben.
}

ching eine zentrale Forschungslücke auf, die es zu schließen gilt.

Aus dem erziehungswissenschaftlichen Kontext ist die aktuelle Veröffentlichung Grammatik des Coachens (Geißler 2017) zu nennen, worin Fragen als die häufigste und eine der wichtigsten und wirkungsvollsten Aktivitäten des Coaches definiert werden. Der „empirischen Rekonstruktion“ liegt ein Verständnis professioneller Coachingprozesse als Handlungssequenzen zugrunde, welche sich aus basalen Handlungseinheiten zusammensetzen, die ihrerseits aus verschiedenen Einzelentscheidungen bestehen, die regelgeleitet miteinander kombiniert werden (vgl. Geißler 2017, S. 1). Die Handlungseinheiten, in Analogie zur Sprechakttheorie von Austin (1962) und Searle (1976) als Sprechakte konzipiert, werden bezüglich ihrer inhaltlichen Proposition und funktionalen Illokution beschrieben. Als zentrales qualitatives Untersuchungsergebnis werden neben vier Typen coachingspezifischer Frageinhalte 11 verschiedene Typen problemlösender Fragen definiert. Basierend auf dem Konzept der individuellen „Kommunikationsentscheidungsmöglicheit" proklamiert der Autor schließlich eine konzeptionelle Systematik von Fragen, die u.a. 816 bzw. 612 idealtypische Frageinhalte von Coach und Klient enthält (ibid, S. 358). Aus gesprächsanalytischer Sicht ergeben sich an dieser Argumentation zwei zentrale Kritikpunkte: Zunächst vertritt der Autor, trotz theoretischer Bezugnahme auf Handlung bzw. Handlungssequenz, eine monologische Sichtweise auf Coaching, in der ein für sich alleine agierender Coach Inhalte kommuniziert. Die hier vorgenommene Illokutionssdifferenzierung bezüglich der Fragen, die auch in der Praxisliteratur weit verbreitet ist, ist als empirische Fundierung nicht haltbar. Die zentralen Eigenschaften von Gesprächen, Sequenzialität und Intersubjektivität, seit den 1970er-Jahre in der Soziologie und der Gesprächsforschung allgemein etabliert, bleiben unberücksichtigt (vgl. u.a. Schegloff 2007; Deppermann 2008; Günthner 2013). Nur diese Sequenzialität garantiert aber die Wirksamkeit von sprachlichem Handeln wie etwa Fragen und damit die Wirksamkeit der Beratungsgespräche. Des Weiteren muss grundsätzlich die Bezugnahme auf die Sprechakttheorie kritisiert werden; diese hat in der linguistischen Pragmatik seit den 1990er-Jahren sehr stark an Bedeutung verloren und wurde selbst in ihren Anfängen nicht für die Analysen von Gesprächen eingesetzt. ${ }^{13}$

Resümierend ist festzuhalten, dass die Forschungslage zu Fragen im Coaching sehr unbefriedigend ist. Neben einigen

\footnotetext{
${ }^{13}$ Es handelt sich um einen sprachphilosophischen, nicht aber einen kommunikativen Ansatz, der durch seinen Fokus auf singuläre Sprechakte der Sprecher_innen nie zum Ziel hatte, die lokalen, sequenziellen und intersubjektiven Aspekte von Gesprächen zu erfassen. Stattdessen ging es darum die sprachphilosophische Basis für den Handlungscharakter von Sprache im Unterschied zur damals vorherrschenden Theorie der Logik zu schaffen.
} 
wenigen Erkenntnisse darüber, dass es sich bei Fragen um eine zentrale Intervention handelt, gibt es noch kein empirisches Wissen über Coaching-spezifische Frage-Sequenzen. Und auch McGee (1999), McGee et al. (2005) sowie James et al. (2010) merken kritisch an, dass ,(w)hile we feel it is helpful to examine question types, it is relevant to note that examining single questions in isolation is overly simplistic due to their impact being a feature of how they are combined and sequenced." Allerdings bleibt es bei ihrer Forderung nach einer sequenziellen Einbettung von Fragen, da sie keine empirischen Studien anhand von authentischem Materials durchführen.

Bezüglich Frage-Sequenzen im Coaching existiert gemäß dem aufgeführten Forschungsstand eine manifeste Forschungslücke, die es zu schließen gilt. Folgende Forschungsfragen sollen dabei helfen:

- Welche Typen von Fragen im Sinne einer linguistischgesprächsanalytischen und institutionsspezifischen Klassifikation finden sich im Coaching und in welchem Verhältnis stehen sie zu den Frage-Typen, die sich in der Coaching-Praxisliteratur finden?

- Wie sind diese retrospektiv und prospektiv in den sequenziellen Ablauf des Coaching-Gesprächs eingebunden? D. h. worauf im Gespräch beziehen sie sich und welche sprachlichen Reaktionen lösen sie aus?

- Welche Typen von Fragen initiieren, prozessieren und beenden die vier Basisaktivitäten im Coaching?

- Gibt es Coaching-spezifische Frage-Kombinationen bzw. Abfolgen von Frage-Typen?

- Wie verändern sich Frage-Sequenzen entlang einzelner Coaching-Sitzungen sowie gesamter Coaching-Prozesse?

Um diese Forschungsfragen in einem nächsten Schritt anhand von empirischem Material, d.h. authentischen Coaching-Gesprächen beantworten zu können, werden im folgenden Abschnitt gesprächsanalytische Erkenntnisse zu Fragen allgemein und im Zusammenhang mit anderen professionellen Hilfe-Formaten wie medizinische oder therapeutische Gespräche zusammengefasst. Diese Erkenntnisse bilden, zusammen mit einer Detaillierung und Berücksichtigung der professionellen Agenden im Coaching, die Ausgangslage der linguistischen-gesprächsanalytischen Forschung.

\section{Frage-Sequenzen im (professionellen) Gespräch - Eine linguistisch-gesprächs- analytische Bestandsaufnahme}

Fragen stellen - neben formulations, (Re-)Interpretationen und extensions - zentrale Gesprächspraktiken in professionellen Hilfe-Gesprächen dar (vgl. Vehviläinen et al. 2008;
Bercelli et al. 2008; Spranz-Fogasy 2010; Mack et al. 2016; Stokoe und Sikveland 2016). Fragen gelten als initiierende bzw. elizitierende Gesprächspraktiken, während die anderen Praktiken als respondierend zusammengefasst werden können (Vehviläinen et al. 2008). Aufgrund ihrer vielfältigen Erscheinungsformen und kommunikativen Verwendungsmöglichkeiten sind Fragen analytisch nicht leicht fassbar (vgl. hierzu z.B. Schegloff 1984, S. 34f; siehe auch Heritage 2010, 2012): sie definieren sich zum einen durch ihre grammatische bzw. morpho-syntaktische Form und zum anderen über ihre pragmatische Funktion. Prosodie ist als zusätzliches Definitionskriterium entscheidend, da so Deklarativsätze wie etwa Das ist Ihre Meinung! von Deklarativsatzfragen wie etwa Das ist Ihre Meinung? unterschieden werden können. Um mit Dayal (2016, S. 1) zu sprechen: „Questions are a quintessential interface phenomenon. They can be studied from the perspective of syntax, semantics, prosody, or pragmatics. Even the basic definition of a question involves a decision between these aspects of grammar."

Während das Zitat von Dayal (2016) auf die sprachlich-formale Ebenen, auf denen Fragen analysiert bzw. beschrieben werden können, fokussiert sowie ihre Funktion thematisiert, beschreiben Tracy und Robles (2009) den endemischen bzw. sinnstiftenden Charakter von Fragen für professionelle Interaktion und deren Beteiligte:

Questioning is one of, if not the, central communicative practice of institutional encounters. As a practice it enacts and reflects an institution's specific goals and values, and the professional and lay identities of key parties (Tracy und Robles 2009, S. 131).

Alle drei Ebenen müssen für eine theoretisch und analytisch fundierte Typologie von Frage-Sequenzen im Führungskräfte-Coaching berücksichtigt werden. Um erneut mit Tracy and Robles (2009, S. 134) zu sprechen: „Besides conceptualizing questions in terms of their form or their function, there is a third way to think about questions and questioning. This third way is to see questioning as a demarcated social practice that exists in particular institutional scenes."

\subsection{Fragen aus formal-linguistischer Sicht}

Auf einer ersten Beschreibungsebene wird folgende morpho-syntaktisch basierte Einteilung der Fragetypen für die Studie vorgenommen (vgl. Spranz-Fogasy 2010; Mack et al. 2016) ${ }^{14}$ :

\footnotetext{
${ }^{14}$ Köller (2004) unterscheidet in Ergänzungsfrage (W-Frage), Entscheidungsfrage (V1-Frage) und Alternativ-Frage (ebenfalls V1-Frage).
} 
- W-Frage; W-Fragen werden eingeleitet durch Interrogativpronomen oder Interrogativadverbien wie etwa wo, wer, wann, was. Diese W-Wörter stehen am Satzanfang der Frage und sind gefolgt von dem Verb in zweiter Satzgliedposition.

- V1-Frage; V1-Fragen bzw. syntaktische Fragen sind gekennzeichnet durch die Erststellung des Verbs. Im Gegensatz zu W-Fragen gelten sie als geschlossen, da die von ihnen erzeugte Antwortoption vordergründig syntaktisch auf Ja/Nein-Antworten (Spranz-Fogasy 2010, S. 50) eingeschränkt ist.

- Deklarativsatz-Frage; Deklarativsatz-Fragen entsprechen in ihrer syntaktischen Struktur einem Deklarativsatz, sind aber z.B. durch ihre Prosodie als Fragesatz gekennzeichnet. D.h., während W-Fragen und V1-Fragen eindeutig durch ihre Form als Frage kategorisiert werden können, gestaltet sich die Kategorisierung bei Deklarativsatz-Fragen komplexer und kann nur durch die Hinzunahme von prosodischen und kontextuellen Informationen gelingen bzw. von den Rezipient_innen so gedeutet werden.

W-Fragen stellen sogenannte offene Ergänzungsfragen dar, bei denen die Antwort nicht bekannt bzw. der Antwortraum offen ist (wenn auch durch die Vorgabe einer kategorialen Ausrichtung des W-Worts nicht komplett offen). Einen Sonderstatus nehmen allerdings Wie-Fragen und Warum-Fragen ein, bei denen „die ins Auge gefassten Informationslücken nicht so klar abgrenzbar sind wie bei anderen Ergänzungsfragen“ (Köller 2004, S. 666). Im Unterschied dazu stellen V1-Fragen und DeklarativsatzFragen geschlossene Entscheidungsfragen dar, die eine Ja- oder Nein-Antwort als type-conforming Antwort (Raymond 2006) erwarten lassen. Da die Praxis-Literatur den Einsatz von geschlossenen Fragen durchgängig kritisch bewertet (Deplazes 2016), sind empirische Erkenntnisse über den tatsächlichen Einfluss geschlossener Fragen auf die thematische und interaktive Entwicklung des CoachingGesprächs dringend notwendig ${ }^{15}$.

Neben dieser rein formalen, morpho-syntaktischen Klassifizierung von Fragen, die eine erste Ebene der analytischen Klassifikation darstellt, unterscheiden Spranz-Fogasy (2005) im Kontext von Arzt-Patient-Gesprächen und Mack et al. (2016) in einer Weiterentwicklung im Kontext von Therapie-Gesprächen des Weiteren zwischen Präzisie-

\footnotetext{
15 Deplazes (2016) hat hierzu bereits folgende empirischen Erkenntnisse geliefert: die Antwort der Klient_innen bei geschlossenen vs. offenen Fragen unterscheidet sich nur unmerklich bezüglich ihrer Länge, thematischer Variabilität und Vielfalt, so dass man nicht davon ausgehen kann, dass geschlossene Fragen weniger anregend und weniger zieldienlich für das Coaching-Gespräch sind als offene Fragen. Siehe auch Heritage (2010) und Spranz-Fogasy (2010), die diese Unterscheidung aus linguistischer Perspektive grundsätzlich problematisieren.
}

rungsfragen und Komplettierungsfragen. In dieser Unterscheidung wird bereits stärker die durch die Frage projizierte Antwort einbezogen und somit eine pragmatischfunktionale Perspektive impliziert: Während erstere (= Präzisierungsfragen) eine direkte Bezugnahme auf das gerade Gesagte darstellen und somit auf der Ebene z.B. der Klient_innen bleiben (= im Sinne eines client-centered behavior), bringen letztere (= Komplettierungsfragen) stärker die Agenda und das Fachwissen der Fragenden, z.B. der Coaches, ins Spiel (= im Sinne eines expert-centered behavior). Es ist eine Unterscheidung, die bei der Gegebenheit eines Wissensgefälles in spezifischen professionellen Interaktionskontexten, wie z.B. auch im Coaching gegeben, ansetzt (vgl. Spranz-Fogasy 2005; Heritage 2010; Mack et al. 2016) und somit auch für die empirische Fundierung von Frage-Sequenzen im Interaktionstyp Coaching relevant ist: Da Coaching, ebenso wie Psychotherapie, auf der verbalen Ko-Konstruktion des Wissens und der Lösung(en) für das Problem der Klient_innen basiert und dabei deren subjektive, lebensweltliche Erfahrungen und Wissensbestände eine zentrale Rolle spielen, die im Rahmen einer Prozess-Beratung re- und ko-konstruiert werden, ist davon auszugehen, dass eine primäre Verwendung von Komplettierungsfragen wie häufig im schulmedizinischen Diagnosegespräch hinderlich wäre (vgl. Mack et al. 2016, S. 29).

Darüber hinaus ist aus den Erkenntnissen aus anderen Interaktionstypen zu projizieren, dass sich der Einsatz von Präzisierungs- und Komplettierungsfragen entlang des Coaching-Gesprächs und in Bezug auf die Basisaktivitäten verändern wird: So ermittelte Spranz-Fogasy (2005) für das Arzt-Patient-Gespräch, dass bei in Gesprächsführung ausgebildeten Ärzt_innen vielfach zunächst Präzisierungsfragen im Vordergrund stehen und Komplettierungsfragen dann nur noch zur Klärung offener Punkte erforderlich werden.

\subsection{Fragen aus funktional-linguistischer Sicht}

Neben den formalen Charakteristika von Fragen sind ihre funktionalen Charakteristika, d.h. die kommunikativen Funktionen, die sie im Gespräch erfüllen, eine zentrale Beschreibungs- bzw. Analysedimension. Die offensichtlichste Funktion von Fragen ist das Einholen von Information basierend auf einem unterschiedlichen Wissenstand der Beteiligten. Allerdings ist dies, zumindest in Alltagsgesprächen, weder ihre häufigste noch wichtigste Funktion (Tracy und Robles 2009, S. 134). Gesprächsteilnehmer_innen stellen mittels Fragen Behauptungen auf, fordern zu etwas auf, laden zu etwas ein, führen Beschwerden aus, fordern das Gegenüber heraus etc. (ibid). Neben diesen Funktionen erfüllen Fragen auch verschiedene beziehungs-orientierte Funktionen, indem sie z.B. Affiliation bzw. Disaffiliation zum Ausdruck bringen oder das Gesicht des Gegenübers 
bedrohen, aber auch bewahren können. Grundsätzlich aber üben Fragen im Gespräch Macht und Kontrolle bezogen auf Inhalt, Gesprächsverlauf respektive Handlungsablauf und Beziehungsgestaltung aus.

Anders in professionellen und institutionellen Gesprächen: Fragen stellen hier ein bzw. das zentrale Instrument des professionellen Handelns von Expert_innen dar. Fragen bzw. als Fragen klassifizierte sprachliche Handlungen beinhalten in diesen Kontexten stets eine epistemische Asymmetrie zwischen Ärzt_innen, Berater_innen, Therapeut_innen oder Coaches und Patient_innen bzw. Klient_innen (cf. Spranz-Fogasy 2010; Heritage 2012; Hayano 2013); dieser Umstand ist im Kontext professioneller und institutioneller Interaktionen von besonderer Bedeutung, bildet doch der unterschiedliche Wissens- bzw. Informationsstand der Beteiligten die Motivation und Eingangsvoraussetzung für derartige Interaktionen (Drew und Heritage 1992; Spranz-Fogasy und Lindtner 2009; Graf und Spranz-Fogasy 2016). Unterschiedliche grammatikalische Gestaltung indiziert dabei unterschiedliche Wissensbestände bei den Beteiligten (Heritage 2010, Spranz-Fogasy 2010). An dieser „Stelle“ überlappen somit äußere Form, d.h. formal-linguistische Aspekte, der semantische Gehalt und in der Folge auch die pragmatische Funktion von Fragenhandlungen ${ }^{16}$. Und während die wichtigste Aufgabe von Fragen auf der thematischen Gesprächs-Ebene Wissens- und Verstehensmanagement ist, sind die wichtigsten Aufgabe auf der Handlungsebene des Gesprächs die Kontrolle der Interaktion sowie die Bearbeitung der Handlungsrationale, also das Voranbringen der professionellen Agenda (Holmes und Chiles 2010). Um mit Hayano (2013, S. 401) zu sprechen: They [questions, EG \& TSF] are employed as a powerful tool in controlling interaction, and they do so by imposing various constraints on answerers (siehe auch Thornborrow 2001, S. 119). Fragen setzen Antworten konditionell relevant und beinhalten dabei eine Präferenz-Organisation für die Gestaltung ihrer second pair parts: „(p)ragmatisch gesehen ist die Frage dadurch eine Frage, dass sie eine Antwort konditionell relevant setzt“" (Mack et al. 2016, S. 29). D.h., erst durch die Antwort, die die Frage strukturell projiziert, erhält die Frage retrospektiv ihre spezielle kommunikative bzw. pragmatische Bestimmung. Die Sprachhandlung Antwort ist beim Stellen der Frage schon angedacht oder impliziert und es

\footnotetext{
16 Allerdings ist die Realität im Gespräch um ein Vielfaches komplexer: so diskutiert Heritage (2012) den Zusammenhang von grammatischer Form und dem Vollziehen bestimmter sozialer Aktionen. Er fokussiert dabei den Unterschied zwischen dem Behaupten von Informationen (assertion information) und dem Fragen nach Informationen (request information) und kommt zu dem Schluss, dass ,(j)ust as requests for information do not require interrogative morphosyntax, so too interrogative morphosyntax does not guarantee that a request for information is in progress." (ibid, S. 3).
}

wird somit, anders als bei anderen Gesprächspraktiken, durch die vorangehende Frage-Handlung eine spezifischere Reaktion eingefordert. Die Antwort als respondierendes Element füllt (oder auch nicht bzw. nur teilweise, siehe unten) die durch die Frage als initiierendes Element eröffnete thematische Informations- oder Wissenslücke. Laut Mack et al. (2016, S. 29) bauen Fragen - im Gegensatz $\mathrm{zu}$ affirmativen Aussagesätzen - sequenzlogisch gesehen eine starke Erwartungshaltung in Bezug auf die projizierte Folgeäußerung auf. Gerade für den professionellen Kontext „Beratung“ (Pick 2017) und spezifischer die Varianten „Prozess-Beratung“ und „Experten-Beratung“ ist eine Unterscheidung in konstruierende, d.h. reflexionsanregende Fragen, die neues Wissen in den Klient_innen hervorbringen, und reproduzierende Fragen, d.h. Informationsfragen, die existierendes Wissen der Klient_innen thematisieren, ein relevantes funktionales Kriterium (vgl. auch Hargie und Dicksons (2004) Unterscheidung in process-questions und recall questions).

Frage-Sequenzen beinhalten somit folgende gesprächsstrukturelle Implikationen: eine sequenzlogische Handlungsstruktur (Fragen setzen Antworten konditional relevant, wobei die Antworten in präferierte und dis-präferierte Antworten unterschieden werden können; Pomerantz und Heritage 2013) und die inhaltslogischen Eigenschaften von Fragen (auch: Präsuppositionseigenschaften von Fragen).

Bezüglich der sequenzlogische Handlungsstruktur von Fragen gilt, dass der erste Teil einer Frage-Antwort-Sequenz den zweiten im Sinne des second pair part eines adjacency pair nicht nur, wie bereits erläutert, einfordert, sondern diesen vorstrukturiert, auch, indem er bestimmte Antwortmöglichkeiten als präferiert und andere als dispräferiert setzt. Beim Phänomen der Präferenz geht es nicht um persönliche Vorlieben der Gesprächsteilnehmer_innen, sondern um strukturelle, handlungslogische Erwartungen im Hinblick auf mögliche Folgereaktionen, hier Antworten. Während präferierte Antwortreaktionen im Sinne der Präferenz für Handlungs- und Darstellungsprogressivität (vgl. Deppermann 2008, S. 232) unmarkiert und umstandslos vollzogen werden, sind dispräferierte Antworten als markierte second pair parts meist durch entsprechende interaktive Phänomene wie Pausen, Reformulierungen oder Korrekturen ausgestaltet (Pomerantz und Heritage 2013). So formuliert Schwitalla (2006, S. 76) z.B. zum Phänomen der Pause:,(z)eitliche Verzögerungen des Einsatzes der eigenen Rede spielen eine wichtige Rolle, um unangenehme Themen oder dispräferierte Antwortzüge gesichtsschonend vorzubringen [...]“. Des Weiteren muss Präferenz bezüglich der second pair parts von Fragen noch in inhaltliche und strukturelle Präferenzorganisation (,type-conforming“ vs. ,type-disconforming“ (Raymond 2003, 2006)) unterschieden werden; ein Umstand, dem schon Schwitalla (1979) Rechnung trägt, indem er verschiedene Grade 
der Responsivität bzgl. der Turnverknüpfung definiert: ,responsiv“" (auf Handlungs- und Themenebene), ,teil-responsiv" (Erwartungen werden nur auf einer der beiden Ebenen erfüllt) sowie „non-responsiv“ (Erwartungen werden auf beiden Ebenen nicht erfüllt) ${ }^{17}$. Die sich in ihrem morphosyntaktischen Aufbau voneinander abhebenden Fragetypen W-Fragen, V1-Fragen und Deklarativsatzfragen unterscheiden sich dabei darin, wie stark sie die Antwort-Gestaltung beschränken (cf. Schegloff 2007, S. 78f), d.h. hier ergibt sich eine erneute Überlappung von formalen und funktionalen Eigenschaften von Fragen bzw. Frage-Sequenzen.

Während der sequenzlogische Aspekt bei Fragen ihren progressiven Einfluss auf den Gesprächsverlauf fokussiert, beziehen sich die inhaltslogischen Eigenschaften von Frage-Sequenzen, d.h. die Präsuppositionen bei Fragen sowie oftmals ihre manifeste Bezugnahme, retrospektiv auf das, was dem Gegenüber durch die fragende Person an Wissen bzw. Information zugeschrieben wird. Da Fragen in der gesprochenen Sprache immer auch Teil interaktiver Sequenzen sind, in denen laufend Wissens- und Verstehensmanagement stattfindet, zeigen sie im Gespräch reziproke Verständniszuschreibungen an (Spranz-Fogasy 2010, S. 53 ff.). So zeigen Boyd und Heritage (2006, S. 154f.) im Kontext von Arzt-Patient Gesprächen z. B., dass Fragen mehr leisten, als Themen und Handlungsaufgaben zu organisieren. Vielmehr enthalten Fragen Präsuppositionen, die die Ärzt_innen als fraglos gegeben behandeln, und Fragen implizieren Präferenzen dafür, welche Erwartungen Ärzt_innen bezüglich des Inhalts der Antwort seitens der Patient_innen haben. D. h., in und mit Fragen wird auch retrospektiv Verstandenes dokumentiert, kommentiert oder bewertet; Fragen können gewissermaßen auch als Stellungnahmen zu ihren Bezugsäußerungen gesehen werden (vgl. Spranz-Fogasy 2010, S. 47). Die bezüglich ihrer morpho-syntaktischen Form unterschiedenen W-Fragen, V1-Fragen und DeklarativsatzFragen implizieren dabei ein zunehmendes Verstehensniveau, das vom Verständnis des gegebenen thematischen Zusammenhangs über die Annahme relevanter Alternativen bis zur Unterstellung einer Proposition reicht (vgl. ausführlich Heritage 2010; Spranz-Fogasy 2010, S. 53 ff).

Fragen als diskursive Praktik sind strukturell asymmetrisch und üben als Instrument der Gesprächssteuerung Macht im Gespräch aus. Bezüglich des unterschiedlichen Wissens- und Verstehensmanagements durch W-Fragen, V1-Fragen und Deklarativsatzfragen ist an dieser Stelle bedeutsam, die Phänomene „Asymmetrie“ und „Expertise“ in ihrer interaktiven Ausgestaltung in Beziehung zu setzen mit der Beschreibung von Coaching als „Dialog auf Augen-

\footnotetext{
17 Siehe auch McMartin (2008) und Stivers (2010) für eine genauere Unterscheidung bezüglich Responsivität und Präferenz: letztere unterscheidet u.a. in „Antwort“ - ,Erwiderung im Sinne einer Nicht-Antwort" - ,keine Antwort".
}

höhe“ und „Coach als Verantwortliche(r) für den Prozess, Klient_in als Verantwortliche(r) für den Inhalt" (vgl. hierzu auch Jautz 2017). Empirische Erkenntnisse zum tatsächlichen Einsatz von Frage-Sequenzen helfen diese zentralen Phänomene differenzierter und handlungsgeleiteter darzustellen.

\subsection{Fragen als interaktionstypspezifisch soziale Handlung}

Schließlich gilt es aus gesprächsanalytischer Sicht, Frage-Sequenzen als institutionsspezifische soziale Handlungen in ihren jeweiligen professionell-institutionellen Gesprächsformaten zu analysieren bzw. zu kategorisieren. Dieser Aspekt geht über die funktional-linguistische Beschreibung von Fragen hinaus, da nun der jeweilige spezifische professionelle Gesprächskontext, in den die Frage-Sequenzen eingebettet sind und den sie gleichzeitig - als zentrale kommunikative Praktik - prägen und hervorbringen, fokussiert wird. Unterschiedliche Handlungsformate bzw. Interaktionstypen (Arzt-Patient Kommunikation, Therapie, Beratung etc.) weisen dabei im Zusammenhang mit Frage-Handlungen interaktionstypische Charakteristika auf. So konstatieren z.B. Tracy und Robles (2009, S. 139), dass „(q)uestions in therapy are considered less direct than questions in medical areas, and may be represented as ways of ,getting the patient talking' rather than getting,facts". "Sie fahren fort, dass ,, (t)herapists try to ask questions from a position of ,not-knowing ' in an attempt to treat the patient as an ,expert' on his or her situation. This is an attempt to limit any a priori institutional methodologies in questionanswer exchanges by making the practice less influenced by a therapist's expertise" (ibid).

Mack et al. (2016) haben für das spezifische therapeutische Format der Operationalisierten Psychodiagnostischen Interviews (OPD) in Anlehnung an Weiste und Peräkyläs (2013) Klassifizierung von formulations, einer weiteren zentralen diskursiven Praktik in therapeutischen Gesprächen, Fragen mit hervorhebender (highlighting) Funktion, mit rephrasing Funktion, mit relocating Funktion und mit exaggerating Funktion empirisch nachgewiesen. Mit rephrasing werden subjektive Anteile von Patientendarstellungen elaboriert, die der Patient nicht benannt oder auch nicht gewusst hat, relocating formulations verknüpfen verschiedene Erzählelemente zu einem Muster und exaggerating formulations überzeichnen die Darstellung von Patienten, sodass sie zum Widersprechen eigener Aussagen gezwungen sind. Darüber hinaus klassifizieren sie lösungsorientierte Fragen und Fragen zur kollaborativen Erklärungsfindung. Und Pawelczyk (2011) beschreibt in ihrer diskursanalytischen Studie der Integrativen Psychotherapie Talk as Therapy eine Frageart, die sie probing questions nennt. Diese weisen - trotz der unterschiedlichen Kontexte 
„Psychotherapiegespräch“ auf der einen und „Arzt-PatientGespräch“ auf der anderen Seite - eine große konzeptuelle Nähe zu den Präzisierungsfragen nach Spranz-Fogasy (2005; siehe auch weiter oben) auf: Beide sind erkennbar auf die vorausgehende Äußerung der Patient_innen bezogen und orientieren sich an dessen Perspektive. Pawelczyk beschreibt probing questions folgendermaßen (2011, S. 67):

The most direct strategy used by the therapists in attempting to access the personal framing of the client's statement is the question "what do you mean". [...] Probes (or probing questions) are follow-up questions that help an interlocutor clarify or expand his/her initial answer [...]. There are numerous places in the clients' narratives that might pose difficulties for the therapist trying to grasp their personal significance for the clients. Moreover, these "veiled" words, phrases or even metaphors often disguise very intimate, private stories that the clients are able to convey at this point of their therapies only in an oblique manner. [...] It also happens that the therapist may have no doubts as to what the client specifically means by a certain phrase, yet he will still confront him/her about its significance. In this way, the client is being gradually instilled with the ability to be aware of the projected emotional states.

Schließlich widmen sich Spranz-Fogasy und Graf (in Vorbereitung a, b) dem Phänomen der expliziten und impliziten Beispielnachfragen im Kontext Operationalisierter Psychodynamische Diagnostik und Coaching. Beipielnachfragen sind definiert als retrospektive Aufforderungen der Therapeut_innen an die Patient_innen, ihre unmittelbar vorausgehende Äußerung mittels konkreter Beispiele zu elaborieren. Erste Ergebnisse bezüglich des Vergleichs von therapeutischem Material (OPD) und Coaching-Daten zeigen, dass sich bei Beispielnachfragen hinsichtlich ihres Veränderungspotentials und ihrer sequenziellen Einbettung nicht die Beispielnachfrage an sich, sondern das der Beispielnachfrage jeweils vorausgehende bzw. nachfolgende Material unterscheidet (Spranz-Fogasy und Graf in Vorbereitung b): Während im therapeutischen Kontext die Therapeut_innen im Vorfeld der Beispielnachfrage die gemeinsame Orientierung auf das Erleben der Patient_innen initiieren, findet diese Orientierung im Coaching durch die Klient_innen selbst statt. Des Weiteren finden sich im therapeutischen Kontext als Antwort oder Erwiderung auf die Beispielnachfrage selten eine Darstellung des Fühlens oder Erlebens bzw. bleibt die Präsentation eines Beispiels ganz aus. Im Unterschied dazu liefern die Klient_innen in den Coaching-Daten bereitwillig(er) Beschreibungen ihrer Wahrnehmung und ihres Erlebens. Bereits hier zeigen sich erste institutionsspezifisch unterschiedliche Funktionen eines bestimmten Typs von Fragen, nämlich Beispielnachfragen; gleichzeitig realisieren diese jeweils unterschiedliche professionelle Agenden in OPD und Coaching (vgl. ausführlich Spranz-Fogasy und Graf in Vorbereitung b).

Über diese ersten, sehr fokussierten Ergebnisse hinaus gibt es noch keinerlei qualitativ-linguistische Erkenntnisse bezüglich Fragen als institutionsspezifische soziale Handlung im Coaching. Das Ermitteln dieser interaktionstypischen Charakteristika von Frage-Sequenzen im Coaching - basierend auf ihren formalen, funktionalen und institutionsspezifischen Besonderheiten - stellt somit ein für die Forschung und die Praxis zentrales Desiderat dar. Interessant sind dabei, neben bereits im Kontext anderer Formate beschriebene Phänomene wie die (quantitative) Verteilung von W-Fragen, V1-Fragen und Deklarativsatzfragen bzgl. ihrer Suchoperationen und Verstehensdokumentationen, der Aneinanderreihung bzw. Abfolge von gleichen und unterschiedlichen Fragentypen innerhalb einer Sequenz, einer Phase, einer Sitzung oder eines Prozesses, die mögliche Veränderung von Fragentypen und ihre Funktionen im Sinne von Bercelli et al. (2013) supra-session courses of action oder aber auch, welche Frage-Sequenzen die Basisaktivitäten „Definieren der Situation“, „Gestalten der Beziehung“, „Ko-konstruieren von Veränderung“ und „Evaluieren des Coachings" initiieren, welche diese prozessieren und welche diese abschließen bzw. welche Frage-Sequenzen in den jeweiligen Basisaktivitäten qualitative und quantitative Besonderheiten aufweisen.

Methodisch passiert eine solche linguistisch-gesprächsanalytische Erforschung von Frage-Sequenzen im Coaching mittels einer Rekonstruktion des moment-by-moment process (Weiste und Peräkylä 2015, S. 4; Peräkylä et al. 2008, S. 12), wobei der sequenzielle Verlauf eines Coaching-Gesprächs Zug um Zug nachvollzogen wird (vgl. Mack et al. 2016). Im Vordergrund steht - im Sinne der unter Punkt 1 beschriebenen Rahmung - die Analyse der sequenziellen Abfolge von turns, die mittels konditioneller Relevanz miteinander verbunden sind. Dadurch wird ein Aufdecken wiederkehrender diskursiver Praktiken und Coaching-spezifischer Handlungsmuster möglich. Fragen müssen in ihrer sequenziellen Organisation als first pair part eines adjacency pair (im Sinne einer Frage-Antwort Sequenz, siehe Fußnote 4) erforscht werden, die prospektiv eine Reaktion des Gegenüber (idealtypisch in formal und funktional als Antwort akzeptierter Form) sequenztechnisch konditionell relevant setzt (Schegloff 2007) und durch die Fragen auch nur retrospektiv eindeutig als Fragen kategorisiert werden können; gleichzeitig muss zur Typologisierung auch der Redebeitrag, auf den sich die Frage retrospektiv bezieht, berücksichtigt werden (ausführlich zur Methode im Zusammenhang mit einer gesprächsanalytischen CoachingProzessforschung siehe Graf (2018); allgemein zum gesprächsanalytischen Vorgehen siehe Becker-Mrotzek und 
Meier 2002; Deppermann 2008; Robinson 2013). Das Ziel dabei ist eine qualitative Beschreibung der kommunikativen Praktik „Fragen“ in ihrer Form und Funktion, wobei neben der Coaching-Funktion, also der institutionellen Funktion, auch auf die Gesprächs-Funktion der Praktik fokussiert werden muss. Daneben gilt es auch die Makrostruktur von Coaching-Gesprächen oder deren overall structural organization (vgl. aktuell hierzu Bercelli et al. (2013) und Mack et al. (2016)) zu analysieren; im Fokus sollte dabei stehen, wie sich Frage-Sequenzen entlang des Coaching-Gesprächs sowie des Coaching-Prozesses verändern. Im Unterschied zum etablierten Phasenmodell im Zusammenhang mit ArztPatient Gesprächen (siehe Heritage und Maynard 2006; Nowak und Spranz-Fogasy 2009), baut die Makrostruktur im Coaching auf kommunikativen Basisaktivitäten auf, die nur bedingt durch ihre zeitliche Abfolge geprägt sind. Diese haben den Referenzrahmen für anzustellende Analysen zu bilden.

\section{Ausblick: Mehrwert einer inter- professionellen und interdisziplinären Erforschung von Frage-Sequenzen im Coaching}

Die qualitativ operierende Gesprächsanalyse erlaubt die Entwicklung und Beschreibung einer Frage-Typologie für das Beratungsformat Coaching auf der Basis einer sequenziellen Analyse der sprachlichen Mikroebene authentischer Coaching-Gespräche. Dieses Vorgehen ermöglicht es die Zug-um-Zug Qualität und Kontextualisierung von FrageSequenzen herauszuarbeiten und $\mathrm{zu}$ beschreiben. Kontextualisierung bezieht sich dabei sowohl auf den lokalen als auch den globalen Gesprächsverlauf im Coaching entlang einzelner Sitzungen und gesamter Prozesse. Auf diese Weise werden Erkenntnisse über die strukturellen und organisatorischen Interaktionseigenschaften von Frage-Sequenzen im Interaktionstyp Coaching generiert. Da der Ansatz in seiner qualitativen Methodik sehr zeitintensiv ist, stellt die erschöpfende Analyse großer Datenmengen eine Herausforderung dar; man fokussiert stattdessen eher den Einzelfall. Nach Schulze et al. (2015, S. 90) sind allerdings ,,... die für die Praxis relevanten und anwendungsbezogenen Erkenntnisse aus einer einzelfallorientierten Gesprächsanalyse auf einer Metaebene nicht gleich ersichtlich." Aus dem Interesse, empirisch fundierte und praxisrelevante Erkenntnisse bezüglich einer Coaching-spezifischen Frage-Typologie zu generieren, ergibt sich an dieser Stelle das Desiderat große Datenmengen analysieren sowie gewonnene Ergebnisse replizieren zu können. Gleichzeitig aber sollte die empirische Fundierung von Frage-Sequenzen nicht an einer beschreibenden und typisierenden ,Stelle“ enden, auch wenn diese auf der Analyse großer Datenmengen basiert. Damit die
Forschungsergebnisse neben theoretischer Relevanz auch praktische Relevanz für die Coaching-Praxis haben, sollte ebenfalls - im Sinne eines zweiten Desiderats - ermittelt werden, wie sich Frage-Praktiken in ihrer sequenziellen Zug-um-Zug-Anordnung entlang des Coaching-Gesprächs verhalten. Da sich die lokale Wirksamkeit von Beratungsgesprächen in bzw. durch Sequenzialität entfaltet (siehe die zahlreichen Veröffentlichung von Peräkylä und Peräkylä et al. im Kontext von Konversationsanalyse und Psychotherapie bzw. anderen Beratungsformate), kann der besondere Beitrag, den Frage-Sequenzen zur angestrebten Veränderung im Coaching und damit zur globalen Wirksamkeit leisten, nur so aufgezeigt werden. Ein solch lokales, den Prozess rekonstruierendes, Wirksamkeitskonzept komplettiert das existierende, globale Wirksamkeitskonzept aus der psychologisch fundierten Wirksamkeits- bzw. OutputForschung (vgl. Graf 2011). So wird ein zentraler Beitrag geleistet zu der von de Haan et al. (2010, S. 11) und anderen geforderten Erweiterung und Vervollständigung des „Was“ im Sinne der Wirkfaktoren um das „Wie“ im Sinne ihrer kommunikativen und interaktiven Ausgestaltung. Der tatsächliche Einfluss und Beitrag von Coaching im Kontext von Lernen und Veränderung kann so ermittelt werden.

Aus beiden Desiderata ergibt sich an dieser Stelle die Notwendigkeit der disziplinären und methodischen Öffnung. Konkret bedarf es der Kombination der wissenschaftlichen Paradigmen der Linguistik und der Psychologie unter Bezugnahme auf sowohl ihre theoretischen als auch empirischen Grundlagen. Gleichzeitig gilt es die professionellen Agenden des spezifischen Beratungsformats Coaching im Sinne einer interprofessionellen Betrachtungsweise und einer gemeinsamen Entwicklung der Forschungsfragen $\mathrm{zu}$ integrieren (vgl. Peräkylä und Vehviläinens (2003) professional stocks of interaction knowledge). Eine empirische Fundierung von Frage-Sequenzen im Coaching, die replizierbare Ergebnisse bezüglich Typen von Fragen und deren Platzierung entlang des Coaching-Gesprächs auf der Basis großer Datenmengen liefert sowie Erkenntnisse darüber generiert, was Frage-Sequenzen im Zusammenhang mit der angestrebten Veränderung der Klient_innen bewirken, kann nur in einer interprofessionellen und interdisziplinären Forschung gelingen.

Grob skizziert bedarf es dafür erstens einer linguistisch-gesprächsanalytisch fundierten Typologie von FrageSequenzen (ermittelt im Rahmen einer case-study und aufbauend auf dem Wissen um die professionellen Agenden im Coaching), auf deren Basis Hypothesen über die Verwendung von Frage-Sequenzen im einzelnen CoachingGespräch und im gesamten Coaching-Prozess generiert werden können. Zweitens bedarf es, aufbauend auf dieser Typologie, der Entwicklung eines Kodierleitfadens, der es ermöglicht, große Datenmengen auszuwerten in Hinblick auf das (sich entlang des Prozesses möglicherweise verän- 
dernde) Auftreten von Frage-Sequenzen. Schließlich bedarf es zusätzlich zu diesem Analyseschritt, dem ein psychologisches Herangehen zugrunde liegt, des Einsatzes eines weiteren psychologischen Instruments, eines Fragebogens zur Messung der Wirksamkeit der ausgewählten und kodierten Coaching-Prozesse. Dies erlaubt es, die lokale, sequenzielle Wirksamkeit von Fragen in Zusammenhang mit der globalen Wirksamkeit von Coaching zu bringen. Abschließend können aus den Erkenntnissen über eine tatsächliche Verwendung und Wirksamkeit von Frage-Sequenzen Handlungsempfehlungen für die Praxis abgeleitet werden, die sowohl in Ausbildungskonzepten als auch in Lehrbüchern zu Coaching Eingang finden.

Ein derartiges interprofessionelles und interdisziplinäres Vorhaben bedarf neben vielem anderen des kritischen Austausches zwischen Coaching-Praxis, Linguistik und Psychologie. Der vorliegende Beitrag möchte dazu den Grundstock legen.

Open Access Dieser Artikel wird unter der Creative Commons Namensnennung 4.0 International Lizenz (http://creativecommons.org/ licenses/by/4.0/deed.de) veröffentlicht, welche die Nutzung, Vervielfältigung, Bearbeitung, Verbreitung und Wiedergabe in jeglichem Medium und Format erlaubt, sofern Sie den/die ursprünglichen Autor(en) und die Quelle ordnungsgemäß nennen, einen Link zur Creative Commons Lizenz beifügen und angeben, ob Änderungen vorgenommen wurden.

\section{Literatur}

Auer, P. (1999). Sprachliche Interaktion. Eine Einführung anhand von 22 Klassikern. Tübingen: Niemeyer.

Austin, J. (1962). How to do things with words. Oxford: Clarendon Press.

Balint, M. (1957). Der Arzt, der Patient und die Krankheit. Stuttgart: Klett.

Becker-Mrotzek, M., \& Meier, Ch (2002). Arbeitsweisen und Standardverfahren der Angewandten Diskursforschung. In G. Brünner, R. Fiehler \& W. Kindt (Hrsg.), Angewandte Diskursforschung (S. 18-45). Opladen: Westdeutscher Verlag.

Bercelli, F., Rossano, F., \& Viaro, M. (2008). Clients' responses to therapists' reinterpretations. In A. Peräkylä, C. Antaki, S. Vehviläinen \& I. Leudar (Hrsg.), Conversation Analysis and Psychotherapy (S. 43-61). Cambridge: Cambridge University Press.

Bercelli, F., Rossano, F., \& Viaro, M. (2013). Supra-session courses of action in psychotherapy. Journal of Pragmatics, 57, 118-137.

Boyd, E., \& Heritage, J. (2006). Taking the history: questioning during comprehensive history-taking. In J. Heritage \& D. W. Maynard (Hrsg.), Communication in medical care: interaction between primary care physicians and patients (S. 151-184). Cambridge: Cambridge University Press.

Braunstein, K., \& Grant, A. (2016). Approaching solutions or avoiding problems? The differential effects of approach and avoidance goals with solution-focused and problem-focused coaching questions. Coaching: An International Journal of Theory, Research and Practice, 9(2), 93-109.

Brock, A., \& Meer, D. (2004). Macht - Hierarchie - Dominanz - A-/ Symmetrie: Begriffliche Überlegungen zur kommunikativen Ungleichheit in institutionelle Gesprächen. Gesprächsforschung Online-Zeitschrift zur verbalen Interaktion, 5, 184-209.

Dayal, V. (2016). Questions. Oxford: Oxford University Press.
Deplazes, S. (2016). KaSyCo - Kategoriensystem zur Analyse von Coachingprozessen. Instrument-Entwicklung und Anwendungsbeispiele. Kassel: Kassel University Press.

Deppermann, A. (2008). Gespräche analysieren. Eine Einführung. Wiesbaden: VS.

Deppermann, A. (2015). Wissen im Gespräch: Voraussetzung und Produkt, Gegenstand und Ressource. InLiSt no, 57, 1-31.

Deppermann, A., Feilke, H., \& Linke, A. (Hrsg.). (2016). Sprachliche und kommunikative Praktiken. Berlin: De Gruyter.

Drew, P., \& Couper-Kuhlen, E. (Hrsg.). (2014). Requesting in social interaction. Amsterdam: John Benjamins.

Drew, P., \& Heritage, J. (Hrsg.). (1992). Talk at work. Cambridge: Cambridge University Press.

Enfield, N., Stivers, T. \& S. Levinson (eds.) (2010). Editorial. Question-response sequences in conversation across ten languages: An introduction. Journal of Pragmatics, 42, 2615-2619.

Fischer-Epe, M. (2012). Coaching: Miteinander Ziele erreichen. Reinbek bei Hamburg: Rowholt.

Freed, A., \& Ehrlich, S. (Hrsg.). (2010). “who do you ask?”. The function of questions in institutional discourse. Oxford: Oxford University Press.

Geißler, H. (2013). Produkt- und Marktsegmentierung von Coaching. In R. Wegener, A. Fritze \& M. Loebbert (Hrsg.), CoachingPraxisfelder. Forschung und Praxis im Dialog - Online-Teil (S. 260-271). Wiesbaden: Springer.

Geißler, H. (2017). Die Grammatik des Coachens. Wiesbaden: Springer.

Graf, E.-M. (2011). Wirksamkeitsforschung und authentische Coaching-Gesprächsdaten: Ist Veränderung im Coaching mittels sprachwissenschaftlicher Methoden analysierbar? In E. Graf, Y. Aksu, I. Pick \& S. Rettinger (Hrsg.), Beratung, Coaching, Supervision: Multidisziplinäre Perspektiven vernetzt (S. 131-146). Wiesbaden: VS

Graf, E.-M. (2015). Kommunikative Basisaktivitäten im CoachingGespräch: Ein linguistischer Beitrag zur Coaching-Prozessforschung. Coaching | Theorie \& Praxis. https://doi.org/10.1365/ s40896-015-0001-1.

Graf, E.-M. (2017). Management Coaching - Ein arbeitsweltliches Beratungsgespräch zwischen Selbst-Reflexion und Output Orientierung? In I. Pick (Hrsg.), Beraten in Interaktion. Eine gesprächslinguistische Typologie des Beratens (S. 77-95). Bern: Peter Lang.

Graf, E.-M. (2018). Äußerung für Äußerung zum Coaching-Prozess: Das Potential einer gesprächsanalytisch fundierten Coaching-Prozessforschung. In R. Wegener, M. Loebbert, A. Fritze \& M. Hänseler (Hrsg.), Coaching-Prozessforschung. Forschung und Praxis im Dialog. Göttingen: Vandenhoeck \& Ruprecht.

Graf, E.-M., \& Spranz-Fogasy, T. (2016). Helfende Berufe - Helfende Interaktionen. In K. Birkner \& N. Janich (Hrsg.), Handbuch Text und Gespräch. Amsterdam: De Gryter.

Graf, E.-M., Sator, M., \& Spranz-Fogasy, T. (Hrsg.). (2014). Discourses of helping professions. Amsterdam: Benjamins.

Graf, E.-M. (submitted). The Discourses of Executive Coaching. Linguistic Insights into Emotionally Intelligent Coaching. Amsterdam: John Benjamins.

Graf, E.-M., Deplazes, S. \& Spranz-Fogasy, T. (in prep.). Questionanswer Sequences in Work-related Coaching.

Grant, A.M. (2012). Making positive change: a randomized study comparing solution-focused vs. problem-focused coaching questions. Journal of Systemic Therapies, 31(2), 21-35.

Grant, A. M., \& O'Connor, S. A. (2010). The differential effects of solution-focused and problem-focused coaching questions: a pilot study with implications for practice. Industrial and Commercial Training, 42(2), 102-111.

Günthner, S. (2013). Ko-Konstruktionen im Gespräch: Zwischen Kollaboration und Konfrontation Erschienen. In U. DausendschönGay, E. Gülich \& U. Krafft (Hrsg.), Ko-Konstruktionen in der In- 
teraktion. Die gemeinsame Arbeit an Äußerungen und anderen sozialen Ereignissen (S. 55-74). Bielefeld: transcript.

De Haan, E., Bertie, C., \& Sills, C. (2010). Critical moments of clients and coaches: a direct-comparison study. International Coaching Psychology Review, 5(2), 109-128.

Hargie, O., \& Dicksons, D. (2004). Skilled interpersonal communication: research, theory, and practice. London: Routledge.

Hayano, K. (2013). Question design in conversation. In J. Sidnell \& T. Stivers (Hrsg.), Handbook of conversation analysis (S. 395-414). Oxford: Wiley-Blackwell.

Heritage, J. (2010). Questioning in medicine. In A. Freed \& S. Ehrlich (Hrsg.), "Who do you ask?". The function of questions in institutional discourse (S. 42-68). Oxford: Oxford University Press.

Heritage, J. (2012). Epistemics in action: action formation and territories of knowledge. Research on Language and Social Interaction, $45,1-29$.

Heritage, J., \& Maynard, D.W. (2006). Communication in medical care. Interaction between primary care physicians and patients. Cambridge: Cambridge University Press.

Holmes, J., \& Chiles, T. (2010). "Is that right?" questions and questioning as control devices in the Workplace. In A. Freed \& S. Ehrlich (Hrsg.), "Who do you ask?". The function of questions in institutional discourse (S. 187-210). Oxford: Oxford University Press.

James, A., Morse, R., \& Howarth, A. (2010). The science and art of asking questions in cognitive therapy. Behavioral Cognitive Psychotherapy, 38(1), 83-93.

Jautz, S. (2017). Immer auf Augenhöhe? Ein Blick in den sprachlichen Werkzeugkoffer im Coaching. In M. Dräger \& M. Kuhnhennn (Hrsg.), Sprache in Rede, Gespräch und Kommunikation. Linguistisches Wissen in der Kommunikationsberatung (S. 47-64). Frankfurt: Peter Lang.

Kallmeyer, W. (2001). Beraten und Betreuen. Zur gesprächsanalytischen Untersuchung von helfenden Interaktionen. Zeitschrift für Qualitative Bildungs-, Beratungs- und Sozialforschung, 2, $227-252$.

Köller, W. (2004). Perspektivität auf Sprache. Zur Struktur von Objektivierungsformen in Bildern, im Denken und in der Sprache. Berlin: De Gruyter.

Konerding, K.-P. (2015). Heilung durch Sprache und Sprechen Linguistik und Psychotherapie. In A. Busch \& T. Spranz-Fogasy (Hrsg.), Handbuch „Sprache in der Medizin“. Handbücher Sprachwissen (hrsg. v. Ekkehard Felder und Andreas Gardt) (Bd. 11, S. 225-242). Berlin: De Gruyter.

Lalouschek, J. (2002). Frage-Antwort Sequenzen im ärztlichen Gespräch. Radolfzell: Verlag für Gesprächsforschung.

Lalouschek, J. (2005). Medizinische Konzepte und ärztliche Gesprächsführung - am Beispiel der psychosomatischen Anamnese. In M. Neises, S. Ditz \& T. Spranz-Fogasy (Hrsg.), Psychosomatische Gesprächsführung in der Frauenheilkunde. Ein interdisziplinärer Ansatz zur verbalen Intervention (S. 48-72). Stuttgart: Wissenschaftliche Verlagsgesellschaft.

Lee, S.-H. (2013). Response design in conversation. In J. Sidnell \& T. Stivers (Hrsg.), Handbook of conversation analysis (S. 415-432). Chichester: Wiley-Blackwell.

Lippmann, E. (2013). Coaching. Angewandte Psychologie für die Beratungspraxis. Berlin: Springer.

Mack, Ch, Nikendei, Ch, Ehrenthal, J. C., \& Spranz-Fogasy, T. (2016). ,[...] hab ich glaub ich die richtigen fragen gestellt“. Therapeutische Fragehandlungen in psychodiagnostischen Gesprächen. OPAL, 3/2016, 1-98.

Mayring, P. (2010). Qualitative Inhaltsanalyse. Grundlagen und Techniken. Weinheim: Beltz.

McGee, D., Del Vento, A., \& Beavin Bavelas, J. (2005). An interactional model of questions as therapeutic interventions. Journal of Marital and Family Therapy, 31(4), 371-384.

McGee, D. (1999). Constructive questions. How do therapeutic questions work? Unpublished doctoral dissertation. Department of Psychology. University of Victoria, Victoria Canada.
McMartin, C. (2008). Resisting optimistic questions in narrative and solution-focused therapy. In A. Peräkylä, C. Antaki, S. Vehviläinen \& I. Leudar (Hrsg.), Conversation analysis of psychotherapy. Cambridge: Cambridge University Press.

Menz, F., Lalouschek, J., Sator, M., \& Wetschanow, K. (2010). Sprechen über Schmerzen. Duisburg: Universitätsverlag Rhein-Ruhr (UVRR).

Miller, K., \& Considine, J. (2009). Communication in the helping professions. In L. Frey \& K. Cissna (Hrsg.), The Routledge handbook of applied communication research (S. 405-428). New York: Routledge.

Mönnich, A. (2004). Gesprächsführung lernen. Welche impliziten Konzeptualisierungen des Kommunikationslernens sind in Methoden zur Entwicklung der Gesprächsfähigkeit zu finden? In M. Becker-Mrotzek \& G. Brünner (Hrsg.), Analyse und Vermittlung von Gesprächskompetenz (S. 87-112). Radolfzell: Verlag für Gesprächsforschung.

Muntigl, P., \& Zabala, L. (2008). Expandable responses: how clients get prompted to say more during psychotherapy. Research on Language and Social Interaction, 41(2), 187-226.

Neipp, M.-C., Beyebach, M., Nuñez, R. M., \& Martínez-González, M.C. (2015). The effect of solution-focused versus problem-focused questions: a replication. Journal of Marital and Family Therapy. https://doi.org/10.1111/jmft.12140.

Newson, G., \& Dent, E. (2011). A work behaviour analysis of executive coaching. International Journal of Evidence Based Coaching and Mentoring, 9(2), 1-22.

Nowak, P. (2010). Eine Systematik der Arzt-Patient-Interaktion. Systemtheoretische Grundlagen, qualitative Synthesemethodik und diskursanalytische Ergebnisse zum sprachlichen Handeln von Ärztinnen und Ärzten. Frankfurt am Main: Peter Lang.

Nowak, P., \& Spranz-Fogasy, T. (2009). Medizinische Kommunikation - Arzt und Patient im Gespräch. In A. Bogner, L. Eichinger, A. Kelletat, H.-J. Krumm, M. Willy, A. Wierlacher \& B. Dengel (Hrsg.), Jahrbuch Deutsch als Fremdsprache 34/2008 (S. 80-96). München: Iudicium.

Pawelczyk, J. (2011). Talk as therapy. Amsterdam: De Gruyter.

Peräkylä, A. (1995). Aids counseling. Institutional interaction and clinical practice. Cambridge: Cambridge University Press.

Peräkylä, A. (2011). After interpretation.: third position utterances in psychoanalysis. Research on Language and Social Interaction, 44(3), 288-316.

Peräkylä, A. (2013). Conversation analysis in psychotherapy. In J. Sidnell \& T. Stivers (Hrsg.), The handbook of conversation analysis (S. 551-574). Chichester: Wiley-Blackwell.

Peräkylä, A., \& Vehviläinen, S. (2003). Conversation analysis and the professional stocks of interactional knowledge. Discourse and Society, 14(6), 727-750.

Peräkylä, A., Antaki, C., Vehviläinen, S., \& Leudar, I. (Hrsg.). (2008). Conversation analysis and psychotherapy. Cambridge: Cambridge University Press.

Pick, I. (Hrsg.). (2017). Beraten in Interaktion. Eine gesprächslinguistische Typologie des Beratens. Frankfurt a.M.: Peter Lang.

Pomerantz, A., \& Heritage, J. (2013). Preference. In J. Sidnell \& T. Stivers (Hrsg.), The handbook of conversation analysis (S. 210-228). Chichester: Wiley-Blackwell.

Radatz, S. (2003). Beratung ohne Ratschlag. Systemisches Coaching für Führungskräfte und BeraterInnen. Wien: Verlag systemisches Management.

Raymond, G. (2003). Grammar and social organization: yes/no Interrogatives and the structure of responding. American Sociological Review, 68, 939-967.

Raymond, G. (2006). Question at work: yes/no type Interrogatives in institutional contexts. In P. Drew, G. Raymond \& D. Weinberg (Hrsg.), Talk and interaction in social research methods (S. 115-134). London: SAGE. 
Robinson, J. (2013). Overall structural organization. In J. Sidnell \& T. Stivers (Hrsg.), The handbook of conversation analysis (S. 257-280). Chichester: Wiley-Blackwell.

Rogers, C. (1951). Client-centered therapy. London: Constable \& Robinson.

Sarangi, S. (2001). On demarcating the space between 'lay expertise' and 'expert laity'. Text, 21(1,2), 3-11.

Sarangi, S. (2010). The spatial and temporal dimensions of reflective questions in genetic counseling. In A. Freed \& S. Ehrlich (Hrsg.), "Who do you ask?" the function of questions in institutional discourse (S. 235-255). Oxford: Oxford University Press.

Scarvaglieri, C. (2013). Nichts anderes als ein Austausch von Worten. Sprachliches Handeln in der Psychotherapie. Berlin: De Gruyter.

Schegloff, E. (1984). On some questions and ambiguities in conversation. In J. Atkinson \& J. Heritage (Hrsg.), Structures of social action: studies in conversation Analyis (S. 28-51). Cambridge: Cambridge University Press.

Schegloff, E. (2007). Sequence organization in interaction: a primer in conversation analysis. Cambridge: Cambridge University Press.

Schein, E. (1969). Process consultation. Reading: Addison-Wesley.

Schmidt-Tanger, M., \& Stahl, T. (2005). Change Talk. Coachen lernen. Coaching-Können bis zur Meisterschaft

Schnurr, S. (2013). Exploring professional communication. Language in action. London: Routledge.

Schreyögg, A. (2010). Ist Coaching reine Prozessberatung oder sind hier auch andere Beratungsmodelle relevant? Zeitschrift für Organisationsberatung, Supervision \& Coaching, 17(2), 119-132.

Schreyögg, A. (2012). Coaching. Eine Einführung für Praxis und Ausbildung. Frankfurt: Campus.

Schulze, H., Reitemeier, U., \& Bialek, J. (2015). Anregungs- und Anwendungspotenzial gesprächsanalytischer Forschung für eine kinderrechtsbasierte Gesprächs- und Beratungspraxis. Eine mikroanalytische Untersuchung über Beteiligung und Befähigung von Kindern in institutionellen. Beratungsgesprächen. Journal für Psychologie, 23(2), 81-112.

Schwitalla, J. (1979). Nonresponsive Antworten. Deutsche Sprache, 7 , 193-211.

Schwitalla, J. (2006). Gesprochenes Deutsch. Eine Einführung. Bonn: Erich Schmidt.

Searle, J. (1976). A classification of illocutionary acts. Language in Society, 5(1), 1-23.

Sidnell, J., \& Stivers, T. (Hrsg.). (2013). Handbook of conversation analysis. Chichester: Wiley-Blackwell.

Spranz-Fogasy, T. (1992). Ärztliche Gesprächsführung - Inhalte und Erfahrungen gesprächsanalytisch fundierter Weiterbildung. In R. Fiehler \& W. Sucharowski (Hrsg.), Kommunikationsberatung und Kommunikationstraining (S. 68-78). Opladen: Verlag für Gesprächsforschung.

Spranz-Fogasy, T. (2005). Kommunikatives Handeln in ärztlichen Gesprächen. In M. Neises, S. Ditz \& T. Spranz-Fogasy (Hrsg.), Psychosomatische Gesprächsführung in der Frauenheilkunde. Ein interdisziplinärer Ansatz zur verbalen Intervention (S. 17-47). Stuttgart: Wissenschaftliche Verlagsgesellschaft.

Spranz-Fogasy, T. (2010). Verstehensdokumentation in der medizinischen Kommunikation: Fragen und Antworten im Arzt-PatientGespräch. In A. Deppermann, U. Reitemeier, R. Schmitt \& T. Spranz-Fogasy (Hrsg.), Verstehen in professionellen Handlungsfeldern (= Schriften zur Deutschen Sprache) (S. 27-116). Tübingen: Gunter Narr.

Spranz-Fogasy, T., \& Lindtner, H. (2009). Fragen und Verstehen - Wissenskonstitution im Gespräch zwischen Arzt und Patient. In E. Felder \& M. Müller (Hrsg.), Wissen durch Sprache. Theorie, Praxis und Erkenntnisinteresse des Forschungsnetzwerks „Sprache und Wissen" (S. 141-170). Berlin: De Gruyter.
Spranz-Fogasy, T., \& E.-M. Graf (in Vorbereitung). Die Bedeutung von Beispielnachfragen im Kontext von Veränderung: Elizitierungs- und Prozessierungstrategien im Vergleich von Therapie und Coaching. In Graf, E.-M., Scarvaglieri, C. \& Spranz-Fogasy, T. (Hrsg.). Veränderungskommunikation in helfenden Berufen. Sprach- und interaktionsanalytische Untersuchungen zu kommunikativen Formen und institutionellen Zwecken. Tübingen: Gunter Narr.

Spranz-Fogasy, T., \& E.-M. Graf (under review). Requesting Examples in Psychodiagnostic Interviews: Therapists' Contribution to the Sequential Co-construction of Clients' Change. In Pawelczyk, J. \& E.-M. Graf (eds.). Understanding Change in Helping Professions. Special Issue for Communication \& Medicine.

Steensig, P., \& Drew, P. (2008). Introduction: questioning and affiliation/ disaffiliation in interaction. Discourse Studies, 10(1), 515.

Stivers, T. (2010). An overview of the question-response system in American English conversation. Journal of Pragmatics, 42, $2772-2781$.

Stivers, T. (2013). Sequence Organizaton. In J. Sidnell \& T. Stivers (Hrsg.), The handbook of conversation analysis (S. 191-209). Chichester: Wiley-Blackwell.

Stivers, T., \& Enfield, N. (2010). A coding scheme for question-response sequences in conversation. Journal of Pragmatics, 42, 2620-2626.

Stokoe, E., \& Sikveland, R. O. (2016). Journal of Pragmatics. Formulating solutions in mediation, 105(6), 101-113.

Theeboom, T., Beersma, B., \& Vianen, A. (2016). The differential effects of solution-focused and problem-focused coaching questions on the affect, attentional control and cognitive flexibility of undergraduate students experiencing study-related stress. Journal of Positive Psychology, 11(5), 460-469.

Thornborrow, J. (2001). Questions, control and the organization of talk in calls to a radio phone-in. Discourse Studies, 3(1), 119-143.

Tiittula, L. (2001). Formen der Gespächssteuerung. In K. Brinker (Hrsg.), Text- und Gesprächslinguistik. Ein internationales Handbuch zeitgenössischer Forschung (Bd. 2(HSK 16.2), S. 1361-1374). Berlin: De Gruyter.

Tracy, K., \& Robles, J. (2009). Questions, questioning, and institutional practices: an introduction. Discourse Studies, 11(2), 131-152.

Tsui, A. (1992). A functional description of questions. In M. Coulthard (Hrsg.), Advances in spoken discourse analysis (S. 89-110). London: Routledge.

Vehviläinen, S., Peräkylä, A., Antaki, C., \& Leudar, I. (2008). A review of conversational practices of psychotherapy. In A. Peräkylä, C. Antaki, S. Vehviläinen \& I. Leudar (Hrsg.), Conversation analysis and psychotherapy (S. 188-197). Cambridge: Cambridge University Press.

Voutilainen, L., Peräkylä, A., \& Ruusuvuori, J. (2011). Therapeutic change in interaction: conversation analysis of a transforming sequence. Psychotherapy Research, 21(3), 348-365.

Wehrle, M. (2012). Die 500 besten Coaching-Fragen. Bonn: managerSeminnare Verlag.

Weiste, E., \& Peräkylä, A. (2013). A comparative conversation analytic study of formulations in psychoanalysis and cognitive psychotherapy. Research on Language and Social Interaction, 46(4), 299-321.

Weiste, E., \& Peräkylä, A. (2015). Therapeutic discourse. In K. Tracy (Hrsg.), The international encyclopedia of language and social interaction (S. 1-10). Oxford: Wiley-Blackwell.

Wilmes, C., \& Loebbert, M. (2013). Coaching als Beratung. In M. Loebbert (Hrsg.), Professional Coaching. Konzepte, Instrumente, Anwendungsfelder (S. 17-48). Stuttgart: Schäffer-Poeschel.

Winkler, O. (2017). Anwendung der Gesprächsanalyse als FeedbackInstrument im Chat-Coaching. Coaching I Theorie \& Praxis. https://doi.org/10.1365/s40896-017-0016-6. 